\title{
Uterine Artery Embolization for Management of Placenta Accreta, a Single-center Experience and Literature Review
}

\begin{abstract}
"Morbidly adherent placenta" is a term that describes the continuum of placenta accreta, increta, and percreta. Placenta accreta is the least invasive form, whereas placenta percreta represents a complete penetration of the trophoblast through the uterus that reaches the serosal surface and potentially invades the bladder, rectal wall, and pelvic vessels. Leaving the placenta in situ in the setting of abnormally invasive placenta is now widely practiced. We herein present three cases of abnormal placental implantation diagnosed by antenatal ultrasound and magnetic resonance imaging, in which uterine artery embolization was performed to induce placental infarction and eventually rapid regression but most importantly to minimize peripartum and postpartum bleeding. As we do this, we sought to review the risks of placenta accreta, increta, and percreta and evaluate the role of endovascular therapy to improve maternal outcomes when abnormal placental implantation occurs.
\end{abstract}

Keywords: Increta, percreta, placenta accrete, uterine artery embolization

\section{Introduction}

The incidence of placenta accreta is 1 per 2500 deliveries. $^{[1]}$ Placenta accreta occurs when there is a defect in the decidua basalis, resulting in abnormal invasion of the placenta. Several factors have been attributed to the increased incidence of placenta accreta resulting in defects in the decidua basalis, such as prior uterine surgery, myomectomy, and curettage in addition to cesarean section deliveries. The maternal mortality rate has been reported as high as $6 \%-7 \%{ }^{[2]}$ The incidence is increasing, and researchers have reported an incidence of 1 in 533 pregnancies in the United States in 2002..$^{[3,4]}$ However, unfortunately, most cases of placenta accreta are discovered at the time of delivery which precipitates severe postpartum hemorrhage due to unsuccessful forcible attempts at removal of the placenta. This leads to major complications including massive blood transfusions, disseminated intravascular coagulopathy, acute renal failure, infectious morbidities, acute respiratory distress syndrome (ARDS), and infertility. Planned cesarean hysterectomy at the time of the cesarean delivery has been the standard of care. ${ }^{[5]}$ Uterine artery embolization (UAE) is an alternative to hysterectomy allowing

This is an open access article distributed under the terms of the Creative Commons Attribution-NonCommercial-ShareAlike 3.0 License, which allows others to remix, tweak, and build upon the work non-commercially, as long as the author is credited and the new creations are licensed under the identical terms.

For reprints contact: reprints@medknow.com conservative management with preservation of the uterus and marked improvement in postpartum hemorrhage. There is insufficient literature comparing standard cesarean hysterectomy to UAE in the management of placenta accreta. Some case loss and significantly better outcomes in conservative treatment while others have shown no benefit. We report three cases of conservative management of antenatal diagnosis of placenta accreta-treated postpartum with UAE and review the literature for placental preservation at the time of cesarean delivery.

\section{Case Reports}

\section{Case 1}

A 34-year-old woman G3P1, with a history of prior cesarean section and dilatation and curettage (D \& C), was admitted to the hospital at 29 weeks for a scheduled cesarean section for removal of a demised fetus. The patient was previously diagnosed by ultrasound at 21 weeks of gestation with placenta previa associated with suspicious placenta accreta. A magnetic resonance imaging (MRI) done showed complete placenta previa complicated by placenta percreta that invaded the urinary bladder [Figure 1]. The patient denied

How to cite this article: Noufaily A, Achou R,
Ashram M, Mokbel M, Dabaj E, Snaifer E, et al. Uterine
artery embolization for management of placenta
accreta, a single-center experience and literature
review. Arab J Intervent Radiol 2017;1:37-42.
reports have shed light on reduced blood

\section{Abdallah Noufaily, Raja Achou, Mitri Ashram, Miziana Mokbel ${ }^{1}$, Emile Dabaj', Elie Snaifer ${ }^{1}$, Abbas A. Chamsuddin}

Departments of Diagnostic and Interventional Radiology, and ${ }^{1}$ Obstetrics and Gynecology Saint George Hospital University Medical Center, University of Balamand, Lebanon

Address for correspondence: Dr. Abbas A. Chamsuddin, Department of Radiology, Saint George University Medical Center, Ashrafieh, Beirut, Lebanon.

E-mail: achamsuddin@yahoo. com

Access this article online Website: www.arabjir.com

Quick Response Code:

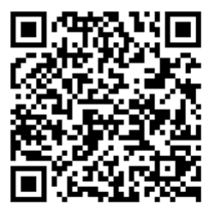


urinary symptoms and vaginal bleeding throughout the course of her pregnancy.

The decision was made to perform bilateral UAE. A left femoral puncture was made with the placement of $5 \mathrm{Fr}$ vascular sheath (Terumo, Somerset, NJ, USA). A 5 Fr Roberts Uterine Catheter (RUC, Cook, Bloomington, IN, USA) was used to cannulate each uterine artery. Successful embolization of both uterine and cystic arteries was performed using 300-500 and 500-700 $\mu \mathrm{m}$ polyvinyl alcohol particle (PVA) particles (Boston Scientific, Huntersville, NC, USA), followed with gelfoam pledgets until stasis [Figure 2].

The patient was then transferred to the operating room where a cesarean section was performed for removal of the demised fetus. The placenta, invading the bladder, was left in situ [Figure 3]. Methotrexate $96 \mathrm{mg}$ IM was given postoperatively, and the patient received 2 units of packed red blood cells (PRBCs).

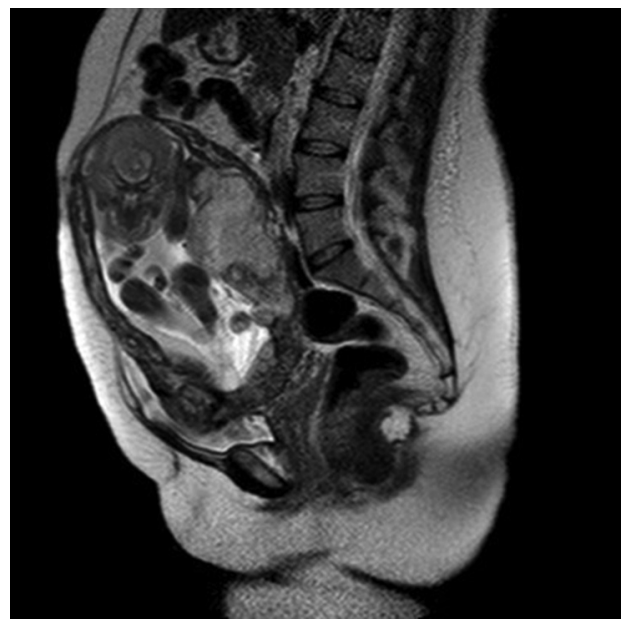

Figure 1: Sagittal T2-weighted prenatal magnetic resonance imaging reveals extensive invasion of the bladder wall by the overlying placenta. Note the demised fetus

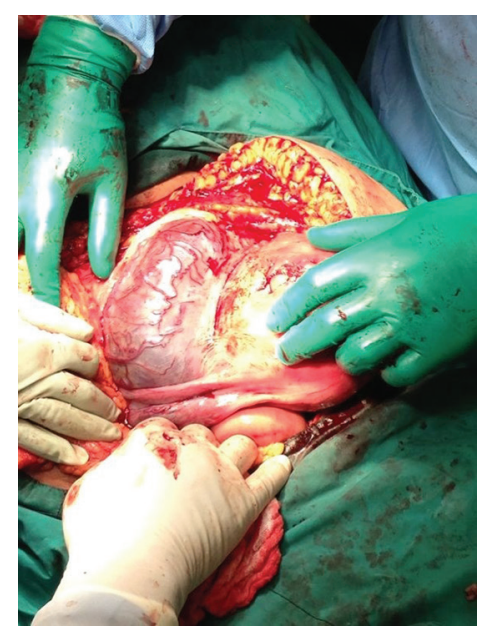

Figure 3: Intraoperative post $\mathrm{C}$-section image showing extensive invasion of the urinary bladder with placental vessels. Note sutures of the $\mathrm{C}$-section in the uterus
Follow up MRI performed at 1 week, and at 3 months, postembolization showed different stages of placental necrosis [Figure $4 \mathrm{a}$ and $\mathrm{b}$ ].

\section{Case 2}

A 30-year-old woman G3P2 at 36 weeks of gestation, with a history of two prior C-sections, presented with profuse vaginal bleeding. The patient was diagnosed antenatally by ultrasound and MRI at 23 weeks and at 30 weeks with complete placenta previa. Follow-up MRI done at 32 weeks showed evidence of complete placenta previa with disruption of the endometrial lining and abnormal penetration of the placental tissue into the myometrium, however, with no definite penetration through the serosa, in favor of placenta increta [Figure 5].

On presentation, the patient was hemodynamically stable. Nonstress test was reassuring, with no evidence of uterine contractions. The decision was made to cannulate the uterine arteries preoperatively in preparation for immediate postpartum embolization.

Bilateral femoral access was performed with the placement of $5 \mathrm{Fr}$ vascular sheath (Terumo, Somerset, NJ, USA), bilateral RUC catheters were placed in a crisscross fashion in the uterine arteries and connected to heparinized saline drips [Figure 6]. The patient was transferred to the operating room.

A cesarean section was performed. A male infant weighing $2700 \mathrm{~g}$ was born with Apgar scores of 7 and 9 at 1 and

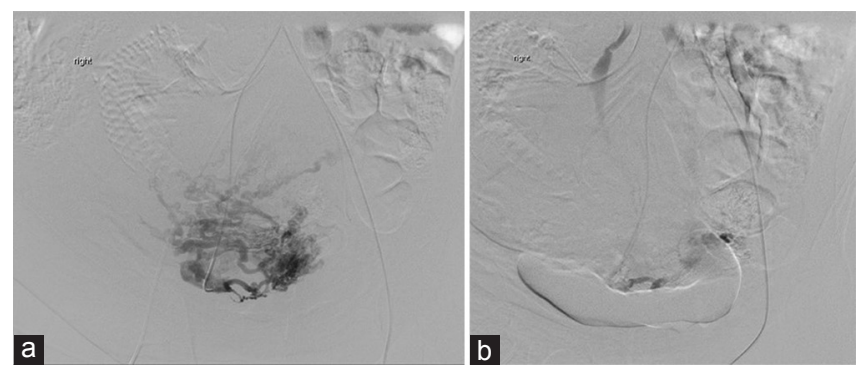

Figure 2: (a) Extensive bladder invasion by the placenta, preembolization. (b) Extensive bladder invasion by the placenta, postembolization

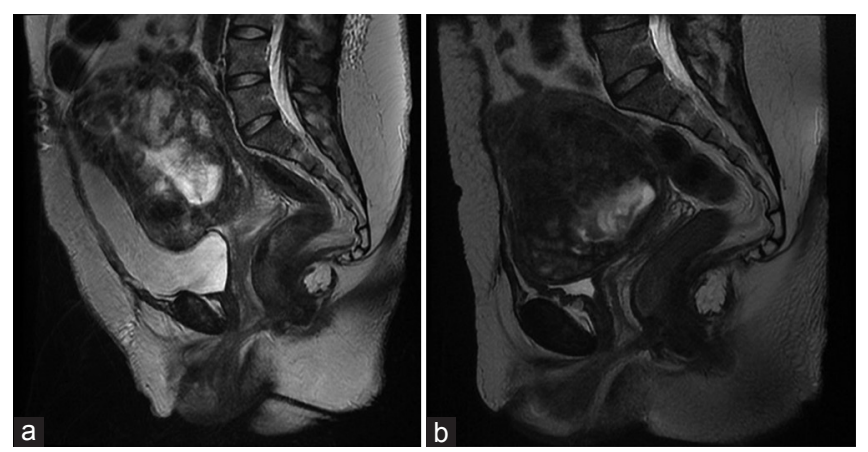

Figure 4: Sagittal T2-weighted images at 1 week (a) and 3 weeks (b) postembolization showing necrosis and progressive atrophy of the placenta 
5 min, respectively. The cord was sutured, and the placenta was left in situ. Then, bilateral UAE was performed using 300-500 and 500-700 $\mu \mathrm{m}$ PVA particles (Boston Scientific, Malborough, MA) followed by gelfoam to stasis [Figure 7]. The patient received 2 units of PRBCs.

Recurrent vaginal bleeding was reported at postoperative day 3. A computed tomography scan done showed retained placental tissue with suspected pseudoaneurysm arising from the left uterine artery [Figure 8]. Repeat arteriography confirmed the presence of abnormal ballooning of a branch of the left uterine artery consistent with a pseudoaneurysm. Embolization was performed with 500-700 and 700-900 $\mu \mathrm{m}$ PVA particles followed with gelfoam slurry until stasis [Figure 9]. The patient eventually stabilized and was discharged home a week after the second embolization. An MRI at 1 week, postdischarge revealed placental infarction with complete atrophy seen on 6 months follow-up [Figure 10].

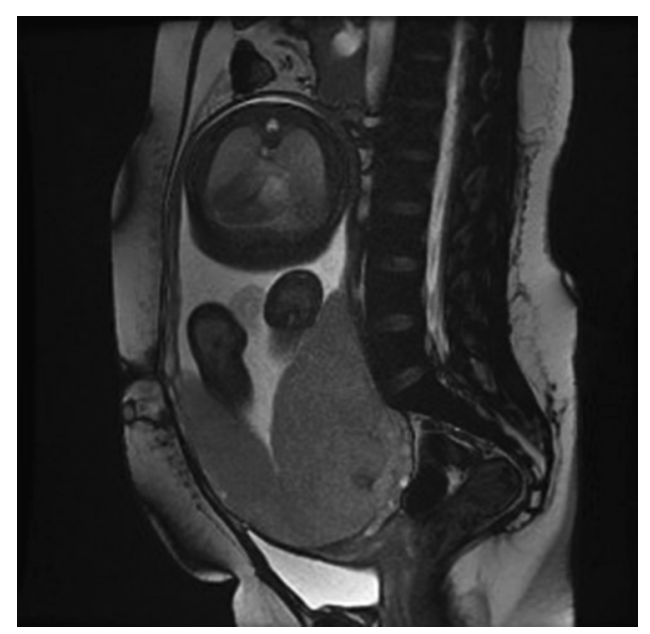

Figure 5: Prepartum sagittal T2-weighted image with complete placenta previa and placental penetration into the myometrium representing placenta increta

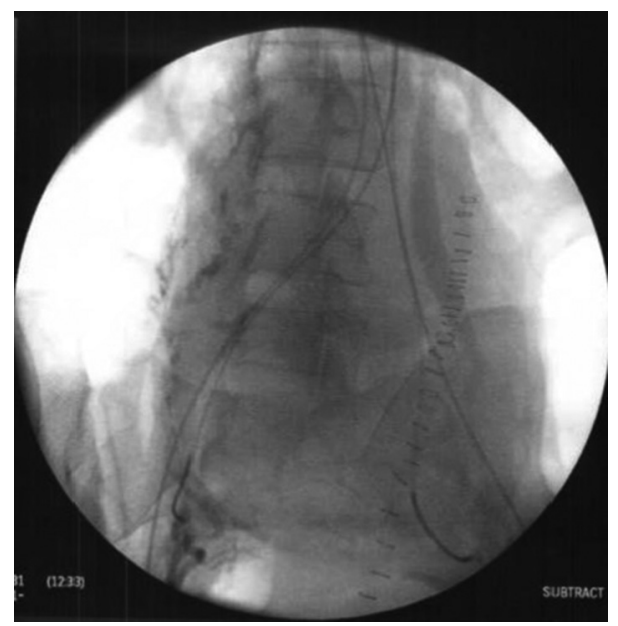

Figure 7: Intraoperative postpartum embolization using crisscrossed Roberts uterine catheter catheters
Case 3

A 32-year-old woman G7P3, with history of three prior cesarean sections and three D \& C, was admitted to the hospital at 36 weeks for profuse vaginal bleeding. The patient was previously diagnosed by ultrasonography, and MRI at 33 weeks showing complete placenta previa complicated by placenta percreta invading the bladder [Figure 11].

Preoperatively, as described under case 2, two RUC catheters were placed in the uterine arteries. A cesarean section was performed. A male infant weighing $2460 \mathrm{~g}$ was born with Apgar scores of 8 and 9 at 1 and $5 \mathrm{~min}$, respectively. The cord was sutured, and the placenta was left in place. Bilateral UAE was then performed using 300-500 and 500-700 $\mu \mathrm{m}$ PVA particles followed with gelfoam pledgets until stasis [Figure 12]. Follow-up pelvic MRI done 6 weeks postembolization showed an atrophic placenta [Figure 13].

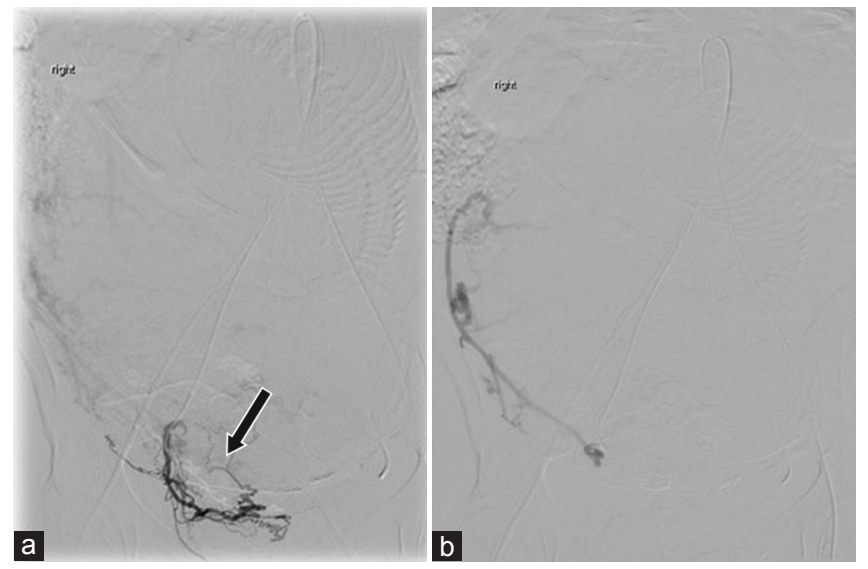

Figure 6: (a) Preoperative uterine arteriogram with contrast pooling in myometrial placental tissue (arrow). (b) Preoperative uterine arteriogram with contrast pooling in myometrial placental tissue

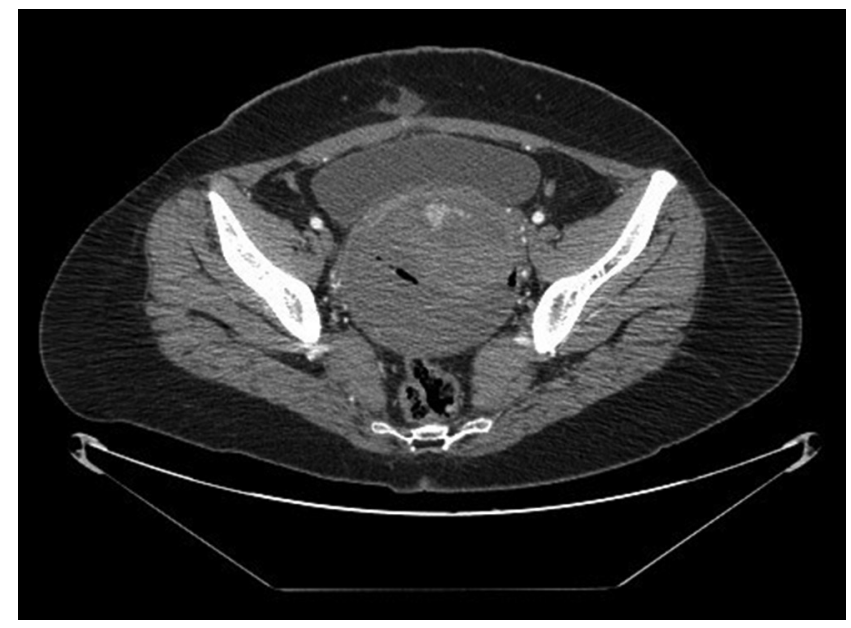

Figure 8: Axial computed tomography scan with findings suggestive of a small pseudoaneurysm arising from a distal branch of the left uterine artery 


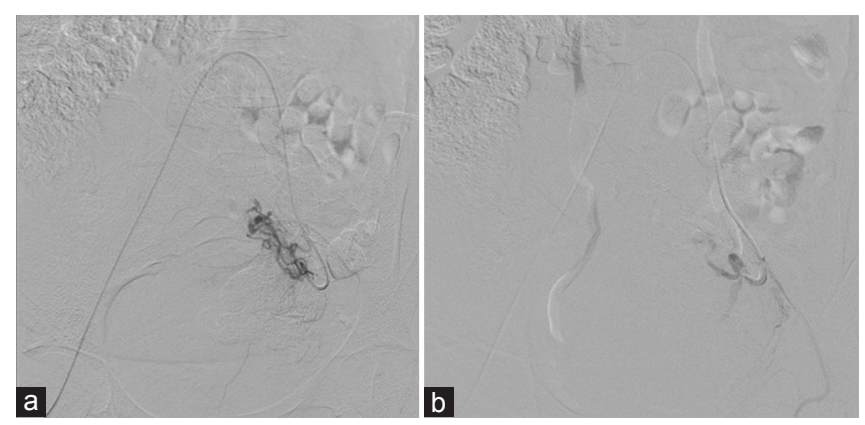

Figure 9: Pre (a) and post (b) repeat embolization of the left uterine artery with distal small pseudoaneurysm
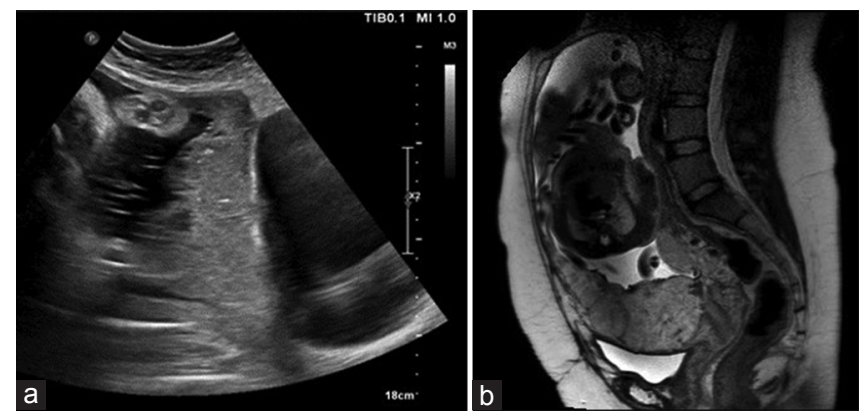

Figure 11: Prenatal ultrasound (a) and magnetic resonance imaging (b) showing complete placenta previa with invasion of the wall of the bladder dome

Postembolization syndrome was seen in all three patients and consisting of pelvic cramps and pain, low-grade fever, nausea, occasional vomiting, and leukocytosis with spontaneous resolution within a week postembolization. No major treatment was needed other than hydration and pain control in addition to wide spectrum antibiotics. Laboratory studies including complete blood count, prothrombin time, partial thromboplastin time, D-Dimer, fibrinogen, and C-reactive protein were requested periodically and were within normal range. All patients were put on prophylactic antibiotics (amoxicillin-clavulanic acid) during their hospital stay.

\section{Discussion}

Placenta accreta occurs when all or part of the placenta attaches abnormally to the myometrium. Three grades of abnormal placental attachment are defined according to the depth of invasion:

- Accreta: Chorionic villi attach to the myometrium rather than being restricted within the decidua basalis

- Increta: Chorionic villi invade into the myometrium

- Percreta: Chorionic villi invade through the myometrium.

In this article, the general term "placenta accreta" will refer to all three grades of abnormal placental attachment (placenta accreta, increta, and percreta) unless otherwise specified. ${ }^{[3]}$

The reported incidence of placenta accreta has increased from approximately 0.8 per 1000 deliveries in the 1980 s
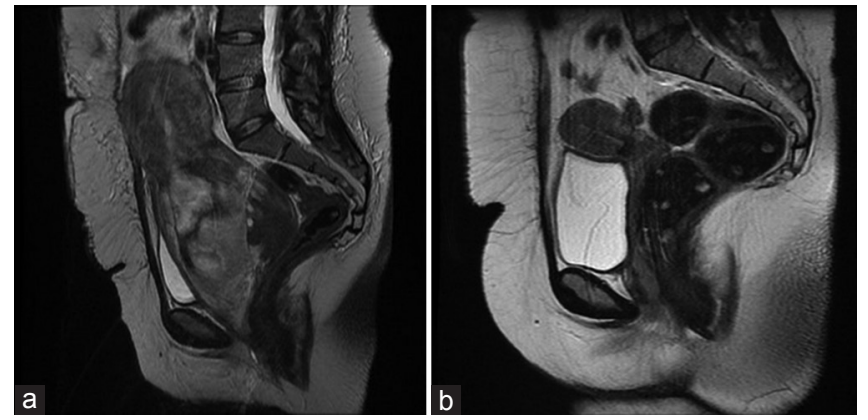

Figure 10: Sagittal T2-weighted images at 1 week (a) and 6 months (b) postembolization with complete placental atrophy
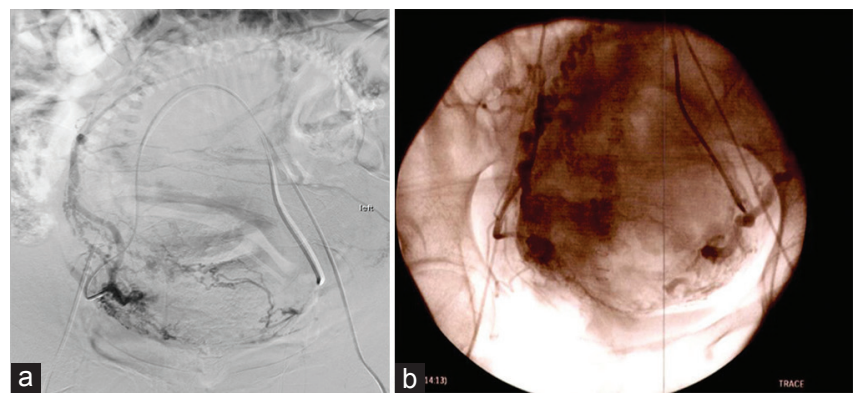

Figure 12: Pre (a) and postpartum (b) angiograms showing bladder invasion by the placenta

to 3 per 1000 deliveries in the past decade..$^{[3]}$ The incidence of placenta accreta in the literature varies between $0.001 \%$ and $0.9 \%$ of deliveries; a rate that depends on what clinical or histopathologic diagnosis is adopted for accreta and the population studied. $70 \%$ of women diagnosed with placenta accreta had previous placenta previa or cesarean sections. ${ }^{[1]}$

An important risk factor for placenta accreta is placenta previa in the presence of a uterine scar. Placenta previa is an independent risk factor for placenta accreta. Additional reported risk factors for placenta accreta include maternal age and multiparity, other prior uterine surgery, prior uterine curettage, uterine irradiation, endometrial ablation, Asherman syndrome, uterine leiomyomata, uterine anomalies, hypertensive disorders of pregnancy, and smoking. ${ }^{[2]}$

Complications of placenta accreta include: (i) Damage to local organs (e.g., bowel, bladder, ureters) and neurovascular structures in the retroperitoneum and lateral pelvic sidewalls from placental implantation and its removal; (ii) postoperative bleeding requiring repeated surgery; (iii) amniotic fluid embolism; (iv) transfusion-related complications (e.g., dilutional coagulopathy, consumptive coagulopathy, acute transfusion reactions, transfusion-associated lung injury, ARDS, and electrolyte abnormalities) from transfusion of large volumes of blood products, crystalloid, and other volume expanders; and (v) postoperative thromboembolism, infection, multisystem organ failure, and maternal death. The exact incidence of maternal mortality related to placenta accreta and its complications is unknown but has been reported to be as high as $6 \%-7 \%$ in case series and surveys. ${ }^{[4]}$ 


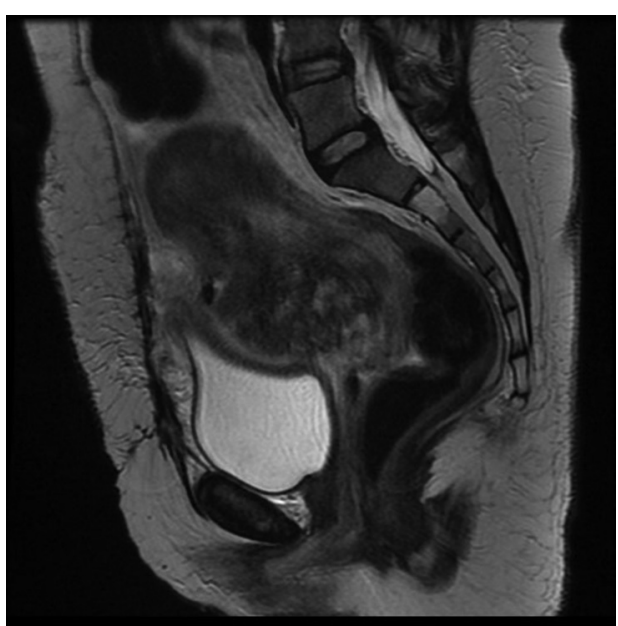

Figure 13: Sagittal T2-weighted images 6 weeks postembolization with placental atrophy and retraction from the bladder wall

When the antepartum diagnosis of placenta accreta is made, it is usually based on ultrasound findings in the second or third trimester. ${ }^{[6]}$ At present, no analyte is considered a necessary component in the workup in women with suspected accreta. Women with a placenta previa overlying a uterine scar should be evaluated for the potential diagnosis of placenta accreta. Women with a placenta previa or "low-lying placenta" overlying a uterine scar early in pregnancy should undergo follow-up imaging in the third trimester with attention to the potential presence of placenta accreta. While obstetric ultrasound is the primary tool for the diagnosis of placenta accreta, MRI can be helpful if ultrasound is inconclusive or if placenta percreta is suspected. ${ }^{[7]}$

When the diagnosis of placenta accreta is suspected antenatally, delivery should be scheduled in an institution with appropriate expertise and facilities including the ability to manage severe hemorrhage. Because the availability of adequate facilities and resources to manage severe hemorrhage at delivery is important, scheduled late preterm delivery (34-35 weeks) is acceptable when placenta accrete is suspected antenatally. ${ }^{[5]}$ The potential need for hysterectomy should be anticipated when the diagnosis of placenta accreta is made. Hysterectomy with the placenta left in situ after delivery of the fetus should be considered. Intraoperatively, attention should be paid to abdominal and vaginal blood loss. Early blood product replacement, with consideration of volume expansion, increasing oxygen-carrying capacity, and normalization of coagulation factors, can reduce perioperative complications. When surgery for placenta accreta is planned, the potential need for postoperative intensive care unit admission should be considered. Pelvic arterial embolization is appropriate for the hemodynamically stable patient with persistent intrapelvic bleeding despite surgical measures. With this regard, it is always important to anticipate this therapy before surgery. We have made it our practice to place uterine arterial catheters before surgery, after which the patient is transferred to the operating room from the angiography suite, and embolization is done intraoperatively. Our small series is promising that this practice would provide safe outcome in these high-risk patients. A hybrid operative suite would be ideal for these cases where catheter placement, delivery, and postpartum embolization could be performed in the same room.

Management of placenta accreta include conservative or surgical approaches. Conservative approach includes leaving the uterus in situ followed by methotrexate, UAE, internal iliac artery ligation or embolization, D \& $\mathrm{C}$ or hysteroscopic loop resection. In contrast, the invasive approach involves immediate hysterectomy. ${ }^{[8-10]}$ Morbidity related to surgery results from extensive blood transfusion, urologic injury, and infections. ${ }^{[11]}$

In the cases mentioned previously, embolization of both uterine arteries was done. This conservative approach has shown to reduce the morbidity of peripartum hysterectomy (including injury to adjacent organs and massive blood transfusion) with potential conservation of fertility. ${ }^{[4,9,12]}$

The conservative approach we describe in this article relies on a multidisciplinary approach that involves the obstetrician, interventional radiologist, anesthetist, urologist and general surgeon in certain cases, readily available blood products, and access to intensive care unit. ${ }^{[6]}$ Conservative management, however, carries increased risk of intrauterine infection, in the setting of embolization, especially with the presence of massive placental infarction. Delayed hemorrhage as seen in case 2 can occur and should be promptly addressed. Therefore, patients must be adequately informed and be aware of the importance of outpatient follow-up for several weeks. ${ }^{[13]}$

\section{Conclusion}

Placenta accreta is a serious condition complicating pregnancy. Surgical management with cesarean hysterectomy is commonly performed. However, conservative management where the placenta is left in situ followed by uterine embolization will conserve the uterus for further pregnancy and help avoid uncontrolled pelvic hemorrhage. Nonetheless, this technique must be used carefully and in appropriately equipped facilities because of the risks associated with severe maternal morbidity.

Our small series highlights the effectiveness of UAE in the management of placenta accreta, reducing the incidence of intraoperative hemorrhage, and postoperational morbidities.

\section{Acknowledgments}

We thank all patients who participated in the study and endured multiple phone calls and follow-ups.

Financial support and sponsorship

Nil. 


\section{Conflicts of interest}

There are no conflicts of interest.

\section{References}

1. Goh WA, Zalud I. Placenta accreta: Diagnosis, management and the molecular biology of the morbidly adherent placenta. J Matern Fetal Neonatal Med 2016;29:1795-800.

2. Fox KA, Shamshirsaz AA, Carusi D, Secord AA, Lee P, Turan OM, et al. Conservative management of morbidly adherent placenta: Expert review. Am J Obstet Gynecol 2015;213:755-60.

3. Placenta Accreta. ACOG Committee Opinion. No. 529. American College of Obstetricians and Gynecologists; July, 2012.

4. Perez-Delboy A, Wright JD. Surgical management of placenta accreta: To leave or remove the placenta? BJOG 2013;121:163-70.

5. Sentilhes L, Goffinet F, Kayem G. Management of placenta accreta. Acta Obstet Gynecol Scand 2013;92:1125-34.

6. Cheung CS, Chan BC. The sonographic appearance and obstetric management of placenta accreta. Int J Womens Health 2012;4:587-94.

7. Levine D, Barnes PD, Edelman RR. Obstetric MR imaging.
Radiology 1999;211:609-17.

8. O'Brien JM, Barton JR, Donaldson ES. The management of placenta percreta: Conservative and operative strategies. Am J Obstet Gynecol 1996;175:1632-8.

9. Alanis M, Hurst BS, Marshburn PB, Matthews ML. Conservative management of placenta increta with selective arterial embolization preserves future fertility and results in a favorable outcome in subsequent pregnancies. Fertil Steril 2006;86:1514. e3-7.

10. Arulkumaran S, Ng CS, Ingemarsson I, Ratnam SS. Medical treatment of placenta accreta with methotrexate. Acta Obstet Gynecol Scand 1986;65:285-6.

11. Doumouchtsis SK, Papageorghiou AT, Arulkumaran S. Systematic review of conservative management of postpartum hemorrhage: What to do when medical treatment fails. Obstet Gynecol Surv 2007;62:540-7.

12. Khan M, Sachdeva P, Arora R, Bhasin S. Conservative management of morbidly adherant placenta - A case report and review of literature. Placenta 2013;34:963-6.

13. Ramoni A, Strobl EM, Tiechl J, Ritter M, Marth C. Conservative management of abnormally invasive placenta: Four case reports. Acta Obstet Gynecol Scand 2013;92:468-71. 